\title{
Une approche sociotechnique des environnements de formation
}

Rationalités, modèles et principes d'action

A sociotechnical approach of training environments: rationale, models and principles for action

\section{Brigitte Albero}

\section{OpenEdition}

Journals

\section{Édition électronique}

URL : https://journals.openedition.org/educationdidactique/715

DOI : 10.4000/educationdidactique.715

ISBN : 978-2-7535-1623-6

ISSN : 2111-4838

\section{Éditeur}

Presses universitaires de Rennes

Édition imprimée

Date de publication : 1 mai 2010

Pagination : 7-24

ISBN : 978-2-7535-1118-7

ISSN : 1956-3485

\section{Référence électronique}

Brigitte Albero, «Une approche sociotechnique des environnements de formation », Éducation et didactique [En ligne], 4-1 | 2010, mis en ligne le 01 avril 2012, consulté le 23 août 2022. URL : http:// journals.openedition.org/educationdidactique/715 ; DOI : https://doi.org/10.4000/ educationdidactique.715 


\title{
UNE APPROCHE SOCIOTECHNIQUE DES ENVIRONNEMENTS DE FORMATION ${ }^{1}$ RATIONALITÉS, MODÈLES ET PRINCIPES D'ACTION
}

\author{
Brigitte Albero (CREAD, Universite Rennes 2 - Haute Bretagne)
}

\begin{abstract}
Résumé : Une étude intégrée des multiples aspects qui déterminent le rôle des objets techniques dans les environnements d'éducation et de formation implique une démarche de type compréhensif, particulièrement difficileà tenir. Dans ce domaine, la recherche s'attache le plus souvent soit aux outils techniques et à la conception des ressources, soit aux usages et aux processus d'apprentissage des sujets. Ce texte s'attache à définir une approche plus large qui puisse rendre compte et des contraintes imposées par les propriétés techniques des objets et de la configuration dynamique de leurs usages par les acteurs en situation. Une première partie aborde la dimension épistémique du problème, en exposant les éléments du cadre théorique et des concepts qui se sont avérés opératoires sur le terrain. La deuxième partie traite de dimensions pragmatiques en analysant l'articulation entre modèles théoriques de l'apprentissage, types de pédagogies et fonctions dévolues aux objets techniques. Une troisième partie aborde la dimension éthique qui conduit à interroger le présupposé de neutralité des technologies dans les pratiques de formation et de recherche, en montrant comment les finalités, les modèles et les valeurs propres aux acteurs orientent et régulent leur activité.
\end{abstract}

Mots-clés : Éducation et formation ; technologies ; pédagogies ; épistémologie de la recherche en sciences humaines et sociales.

Brigitte Albero

Depuis l'ardoise jusqu'aux plateformes numériques, l'action éducative a toujours pris appui sur les moyens techniques du moment. Habituellement décrits en termes seconds (supports, outils ou auxiliaires), ces objets sont perçus de manière strictement fonctionnelle, dissociée non seulement de leur « mode d'existence » spécifique en tant qu'objets techniques (Simondon, 1958), mais aussi de l'activité des utilisateurs et de leur rôle moteur dans l'évolution des sociétés. Aujourd'hui, l'intégration du numérique dans tous les domaines rend cette dissociation plus que jamais inadéquate. Elle contraint à inventer d'autres cadres d'analyse qui tiennent compte des nouvelles formes de relations et d'interdépendance entre objets techniques et activité humaine. Le secteur de la formation, en particulier, ne peut plus ignorer le changement radical des modèles d'organisation et de fonctionnement des environnements, ni l'apparition de systèmes hybrides (et techniques et sociaux) qui transforment profondément nos rapports au monde et à l'action. Dans cette perspective, l'enjeu est double: comprendre l'évolution d'environnements de formation toujours plus complexes et fournir des orientations à l'analyse et à l'intervention dans ce domaine.

Dans sa première partie, cette contribution présente les éléments d'un cadre théorique et d'un mode d'analyse capable de prendre en compte l'évolution des conditions de la formation. Dans une deuxième partie, divers modèles sous-jacents aux pratiques sont identifiés et revus à la lumière des propositions présentées. La troisième partie explicite un certain nombre de constats et d'interrogations visant à relier les aspects conceptuels et pratiques, techniques et éthiques dans ce champ.

\section{Renouveler l'approche des objets techniques en formation: fondements théoriques}

Le constat du caractère émietté et non cumulatif des études sur les environnements de formation médiatisés (Albero, 2004) confirme la nécessité d'un renouvellement du cadre et des méthodes d'analyse dans ce domaine. 


\section{Les mots en usage: trace sémantique des persis- tances et des changements}

La terminologie qui se réfère aux objets techniques n'est pas neutre. Elle renvoie non seulement aux changements de représentations associées à l'innovation et à la « logique d'usage » (Perriault, 1989), mais aussi aux évolutions des pratiques dès lors qu'elles tendent vers une « hybridation $»$ (Perriault, 1996) des modèles. Elle peut témoigner d'un basculement radical proche du changement de paradigme conceptualisé en épistémologie des sciences par T. Kuhn (1962).

Dans le champ des pratiques, la décennie 198090 voit la classification des outils selon leur support technique (papier, audio, audiovisuel, numérique) et le médium utilisé (livre, radio, télévision, vidéo, web) s'effacer au profit du terme "technologies $»^{2}$. Cette évolution marque un tournant. Elle exprime de manière sous-jacente plusieurs aspects à la fois: une dynamique d'intégration des diverses techniques en une seule entité polymorphe; selon une acception étymologique, une relation organique entre la technè relative aux outils et savoir-faire spécifiques et le logos en tant que connaissance rationnelle; dans l'acception portée par l'anglicisme, un lien rarement explicité avec la dimension industrielle et économique de production et d'exploitation des objets. La sémantique des modalités de formation évolue parallèlement, passant progressivement ${ }^{3}$ d'une centration sur les objectifs et l'action de l'instance de formation à une centration sur l'activité de l'apprenant.

Parallèlement, dans ce domaine fortement investi de croyances et d'intérêts divers, la tendance des travaux de recherche à employer les mêmes termes que ceux des acteurs dans leurs pratiques sociales rend parfois difficile la distinction entre les postures d'investigation, d'expertise scientifique et/ou professionnelle ou encore d'intervention militante.

Dans ce contexte, il est apparu nécessaire d'engager la réflexion sur deux plans: élaborer un cadre épistémique susceptible de produire une approche théorique exprimée par une sémantique moins ambiguë; en dégager des concepts opératoires susceptibles de rendre compte des pratiques de formation et de leurs relations avec les objets techniques, quelle qu'en soit la période historique, la culture de référence ou l'idéologie sous-jacente.

\section{La perspective sociotechnique en sciences humaines et sociales}

Une lecture transversale des approches de l'activité technique dans diverses disciplines de SHS (Albero, 2010a) a permis de relier les travaux déjà anciens d'anthropologues (Mauss, Leroi-Gourhan, Haudricourt), d'historiens (Parain, Gille, Jacomy) et de sociologues (Scardigli, Flichy, Gras) à des travaux plus récents dans le domaine des sciences et des techniques (Akrich, Callon, Latour). Leurs apports respectifs peuvent ainsi être réunis autour de la définition d'une approche sociotechnique fondée sur le concept de couplage entre technique et activité humaine. Ces travaux qui se concentrent autant sur l'activité et les relations sociales que sur les artefacts qui en sont les supports et les moyens, mettent en valeur le lien indissociable entre fonction et usage. Loin d'être disjoints du corps social, les objets techniques en font partie et y participent directement par tout un ensemble de processus: depuis leur production, leur diffusion, leurs usages jusqu'aux symboles dont ils sont porteurs. Ces travaux spécifiques aux SHS mettent particulièrement en lumière la manière dont l'objet technique mobilise en les matérialisant toutes les dimensions de l'activité humaine - politique, économique, culturelle, sociale, artistique - ainsi que leurs enjeux d'utilité et d'efficacité, de pouvoir et de prestige, mais aussi d'éthique et d'esthétique.

En intervenant toujours plus avant dans les processus ordinaires de l'action et de la connaissance, les technologies contemporaines ne cessent de renforcer ce couplage. Dans ce contexte, on propose de voir en quoi, appliquée aux environnements de formation, la perspective sociotechnique est susceptible de renouveler les points de vue et les modèles d'analyse, en proposant d'autres modes de compréhension des phénomènes et d'autres principes d'action.

\section{Objets techniques et environnements de formation: de la disjonction au couplage}

Comme dans les autres secteurs d'activité, la formation commence par considérer les objets techniques d'abord d'un point de vue utilitaire, selon les besoins, moyens et modèles de référence, plus ou moins partagés, de manière explicite ou non, par les différents acteurs. Elle les met cependant en œuvre 
dans un domaine très particulier - l'éducatif - déterminé par des institutions, des règles, des valeurs et des visées spécifiques qui la soustraient, en partie, aux logiques strictement économiques.

Analyser les environnements de formation dans une perspective sociotechnique aboutit à en modifier profondément le cadre d'analyse. Le changement de point de vue qui contraint à prendre en compte les relations de dépendance entre activité, technique et environnement mène à des conceptions systémiques plus écologiques, au sens de G. Bateson (1977), centrées davantage sur les interactions entre éléments que sur leurs caractéristiques individuelles. Dans ce cas, les objets techniques ne sont pas perçus comme disjoints de l'action de formation, ni des spécificités de ses contextes culturels et institutionnels. En tant qu'instruments cognitifs, ils matérialisent des modèles épistémiques et idéologiques particuliers et sont analysés dans la dynamique de leur interaction avec les acteurs.

\section{Dispositifs, instrumentation et configuration : trois concepts opératoires en relation}

Les perspectives ouvertes par l'approche sociotechnique ont permis de dégager trois concepts opératoires, cohérents avec l'analyse sémantique de termes courants et avec les observations issues de plusieurs études conduites dans le domaine (Albero, $1998 ; 2003 a / b)$.

\section{Le dispositif de formation}

Dans le sens courant du terme, un dispositif est une organisation rationnelle de moyens matériels et humains selon un ensemble cohérent de buts et d'objectifs en vue d'un résultat précis. L'emploi de ce terme en formation a largement servi une conception fonctionnelle de l'action, indépendante en grande partie des caractéristiques portées par les acteurs. Dans la continuité de travaux antérieurs (Linard, 1989; Jacquinot-Delaunay, Monnoyer, 1999), la perspective sociotechnique permet de prendre en compte davantage les phénomènes de construction interdépendante de sujets et d'objets, déterminée par les projets et modèles des concepteurs, mais aussi par celui des utilisateurs et par les possibilités techniques qui, en pré-scrivant (Akrich, 1987) des normes et des procédures particulières d'action: «imposent toujours des structures et des rapports nouveaux » (Linard, 1989, p. 16).

Ainsi perçu, le dispositif en formation devient le lieu où s'actualise un jeu permanent de tensions entre les grands axes directeurs de l'action: le passé à partir duquel il se construit; les anticipations sur son avenir possible qui orientent son développement; les finalités qui lui donnent sens et permettent de sélectionner les objectifs et buts poursuivis; les contingences enfin avec lesquelles il faut composer en permanence. De ce jeu, émerge un autre couple de tensions qui affecte de manière visible et déterminante le fonctionnement: l'historicité du dispositif qui prend appui sur l'interprétation que les acteurs font du passé et sa temporalité inscrite dans les aléas du quotidien, le déroulement effectif, des projets et la production pratique des résultats (Albero 1998; 1999). Quand le dispositif intègre ces divers éléments comme sources de pilotage de l'action, dans des boucles de retour d'information qui permettent de corriger et réguler à mesure son fonctionnement, il change de nature. Il enclenche des processus d'auto-organisation qui le font accéder à une dimension supérieure d'interaction dynamique, de type biologique, qui lui permet de gagner en capacité d'adaptation et d'évolution.

\section{Une approche ternaire}

Des observations allant dans ce sens ont conduit à proposer une approche ternaire des dispositifs de formation (Albero, 2010b/c) qui permet de comprendre et d'analyser de façon plus précise nombre de phénomènes constatés de manière empirique. Trois dimensions constitutives sont distinguées: l'idéel, le fonctionnel de référence et le vécu. Le dispositif idéel est l'ensemble des idées, principes, modèles et valeurs qui orientent et structurent les décisions, actes et discours des acteurs au cours du projet. Il appartient pour l'essentiel au dessein des concepteurs - à la fois visée idéale et concept directeur - fil rouge qui relie les structures, les interactions et les phases de réalisation. Le dispositif fonctionnel de référence est la mise en actes pratique de l'idéel, le projet opérationnel explicité dans les discours, les textes et documents (définitions, planifications, étapes, évaluations). Il constitue le pivot central de l'ingénierie qui organise, contrôle et régule 
le fonctionnement global, la référence pragmatique normative qui permet d'évaluer les résultats, non seulement par rapport au dispositif idéel, mais aussi aux réalités pédagogiques, économiques et matérielles du moment. Il doit également composer avec les dimensions socio-émotionnelles du dispositif vécu, tel qu'il est expérimenté au quotidien. Le dispositif vécu correspond à l'expérience personnelle des différents acteurs (décideurs, enseignants, techniciens, étudiants) et à l'aménagement continu qu'ils réalisent: en fonction de leur histoire, de leurs aspirations et dispositions propres, mais également de leurs statuts et de leurs rôles, ainsi que des formes d'interactions et de négociations et des tensions auxquelles ils sont confrontés au jour le jour.

Cette conceptualisation permet d'analyser le fonctionnement des dispositifs en tant qu'entités sociotechniques complexes: évolutives, polymorphes, en partie imprévisibles, résultats fluctuants de l'interaction entre leurs trois dimensions constitutives. Chaque dispositif devient ainsi une organisation spécifique, située dans un espace-temps précis, fonction d'un modèle stratégique particulier d'intervention et de transformation sociocognitive, constamment soumis aux résultats en retour de son propre fonctionnement. Il n'a pas la même apparence selon que l'on s'intéresse au projet idéel qui l'oriente, à l'architecture et à l'ingénierie fonctionnelle qui le charpentent ou à l'activité pratique qui le réalise. Globalement, il est à la fois la somme de toutes ces dimensions et leur émergence, ce quelque chose de plus, largement imprévisible et bien connu des acteurs, qui fait que "ça marche » ou bien au contraire que «ça bloque » et que « rien ne va plus».

Toutefois, les dimensions, descripteurs et indicateurs dégagés ne concernent que les niveaux macro et médian de l'organisation des formations. Ils ne permettent pas d'appréhender le micro niveau de l'activité effective des acteurs. Les concepts d'instrumentation des apprentissages et de configuration d'activité apportent les moyens d'une analyse plus précise sur ce plan.

\section{Linstrumentation des apprentissages}

Faisant suite à divers travaux en psychologie $\mathrm{du}$ travail d'origine russe et finlandaise, l'approche anthropocentrée de l'activité instrumentée développée en France par P. Rabardel (1995) a renouvelé les études dans le domaine. En intégrant à la définition technocentrée de l'instrument les schèmes mentaux d'utilisation de l'usager, le concept déplace l'attention de l'objet en soi à la relation de l'utilisateur avec cet objet. En tant que couplage artefact-schèmes d'utilisation, l'instrument participe à la fois du sujet et de l'objet et, par l'activité qui les relie, de l'environnement naturel et social des usagers. Cette redéfinition de l'instrument s'avère particulièrement pertinente en formation. Elle permet au chercheur d'élargir son point de vue, non seulement à tous les objets matériels ou symboliques qui entrent dans le déroulement de l'activité, mais aussi à leurs interactions. Les médias, technologies, matériaux didactiques et pédagogiques, outils méthodologiques et techniques professionnelles ne sont plus des objets juxtaposés. Ils deviennent significatifs et interdépendants par rapport à l'action de l'acteur. On comprend alors pourquoi l'artefact ${ }^{4}$, externe au sujet, ne devient effectivement instrument ${ }^{5}$ que lorsqu'il est intériorisé et approprié par lui - actualisé, transformé ou détourné - en fonction de ses schèmes internes, ceux-ci étant eux-mêmes le produit constamment remanié de ses acquisitions culturelles - familiales, scolaires, professionnelles - et de ses expériences antérieures. Dans ce cadre, l'instrument n'est plus considéré comme un simple auxiliaire, mais comme une composante fonctionnelle de l'action.

Définir la relation pédagogique comme une modalité particulière d'interactions instrumentées, à la fois médiatisée par une technique et médiée par une relation humaine, permet d'élargir le cadre de l'analyse au plan de la recherche et d'envisager des systèmes de formation plus ouverts, adaptatifs et intégrés au plan des pratiques.

Sur ce point, la différenciation entre médiatisation et médiation proposée par M. Linard (1989) est toujours pertinente, même si elle est à nuancer. Pour ce chercheur, la « médiatisation » renvoie à la transposition technique du message d'un medium à un autre et à sa transformation par les caractéristiques particulières du support. Les systèmes symboliques et les procédures techniques utilisés pour traiter le même savoir - support papier, audio, vidéo, numérique - ne sélectionnent et ne traduisent ni les mêmes aspects, ni les mêmes modes de présentation et de 
structuration de la perception et du raisonnement. La médiatisation est ainsi propre au traitement technique des messages et aux processus cognitifs qu'il engage chez l'usager. La " médiation » serait plutôt le propre de l'intervention humaine, par sa prise en compte, non seulement de la forme et du sens des messages, mais également de ses conditions humaines de production: interlocuteurs, contextes, situations et multiples aléas de la communication (différences interindividuelles, difficultés cognitives, sociales et relationnelles, accidents et imprévus, etc.). Dans des travaux sur la télévision appuyés sur les recherches d'U. Neisser (1967) et de G. Salomon (1993), C. Compte (1985a/b) a montré comment par sa spécificité technique, le média peut également jouer un rôle de "médiation cognitive ». Dans ce cas, le langage symbolique structuré par la « rhétorique télévisuelle » (ibid., 1998) et décodé consciemment ou non par l'usager, peut conduire à une évolution de ses représentations et de ses schèmes mentaux. Le processus de "facilitation » (Marks Greenfield, 1984), porté par le médium et l'aide à l'acquisition cognitive qui en résulte, peut être considéré comme une forme implicite de médiation. La différence entre médiatisation et médiation tiendrait ainsi moins à l'opposition entre intervention humaine et support technique qu'à la nature du processus en jeu: la médiatisation, simple transposition d'information d'un support à un autre; la médiation, accompagnement de l'activité de transformation de l'information en connaissance de la part des apprenants ${ }^{6}$.

En formation, l'acte d'instrumenter ne peut donc se réduire à la mise à disposition des outils techniques $^{7}$. Elle consiste à donner également aux étudiants, considérés comme auteurs et acteurs de leur apprentissage, les moyens de construire l'outillage cognitif sensorimoteur, perceptif, conceptuel - et la dynamique psychologique d'auto-développement grâce auxquels ils pourront être placés en situation de réussite. Le concept d'instrumentation devient ainsi opératoire (Albero, 1998, 2003b). Il rejoint les perspectives vygotskiennes pour lesquelles les instruments sont, au même titre que la langue, un donné autant socioculturel - orienté vers le psychisme et la conduite du sujet - que technique - orienté vers l'objet, qu'il soit savoir ou machine -. Dans cette acception, il devient possible de penser l'objet technique comme un artefact qui à la fois médiatise certains processus cognitifs chez le sujet en les matérialisant et les médie en les facilitant. C'est à ces conditions qu'il est possible de parler d'une instrumentation globale des apprentissages qui peut alors se décliner en sept grands domaines: technique, informationnel, méthodologique, social, cognitif, métacognitif, psycho-affectif (Albero, 2003a).

\section{Les configurations d'activité}

Le concept éliasien de configuration offre un moyen heuristique d'identifier les formes d'appropriation du dispositif par les acteurs et ses modes d'instrumentation, ces formes évoluant mais restant relativement stables à l'intérieur de la variété des possibles. Ce concept présente l'avantage de s'inscrire dans le même paradigme que celui de dispositif (Albero, 2003a). C'est une construction intermédiaire entre les perspectives globale et située, intégrative et distribuée, générique et singulière de l'action. Comme le concept de dispositif, il permet d'articuler différents niveaux d'analyse selon une même logique et de dégager des constantes, des idéaux-types et des formes dominantes à l'intérieur d'une grande variété de combinaisons possibles.

Les travaux concernant l'analyse de l'activité en éducation et formation (Barbier, 1996; 2000; Barbier, Durand, 2006) utilisent le concept de « configurations sociales d'apprentissage » (Zeitler, 2006) ou de « configurations d'activité » pour désigner l'identification de ces «formes dynamiques qui conservent une certaine stabilité, organisation, signification » (Durand, Saury, Sève, 2006, p. 76). En rupture avec la conception qui réduit l'activité à « l'exécution simple d'un prescrit » (ibid., p. 68), ce concept permet de prendre en compte la manière pratique par laquelle les acteurs (responsables, intervenants, étudiants) s'approprient et sélectionnent un ensemble de possibles dans l'environnement potentiel du dispositif. Cette actualisation différentielle des possibles s'observe quand on étudie le détail des configurations de l'activité effective des individus (Roublot, 2010). À la fois singulières et partagées (Zeitler, 2006; 2007), les configurations pratiques apparaissent souvent très éloignées et de l'idéel et du fonctionnel de référence proposés par les instances de formation.

Cette approche met en évidence les liens transversaux d'interdépendance permanente entre les trois dimensions (idéel, fonctionnel, vécu). Elle permet d'étudier les niveaux médians entre social et 
individuel et de traiter la multiplicité des vécus d'acteurs comme une émergence supérieure à la juxtaposition des vécus singuliers. Elle montre aussi la part déterminante de la dimension individuelle dans l'efficacité finale du dispositif mesurée en termes d'apprentissage. C'est à ce stade d'analyse qu'apparaissent les tensions entre la description objective du dispositif fonctionnel et son appropriation plurielle par les acteurs. On découvre qu'il existe autant de dispositifs vécus que de sujets, chacun actualisant à sa manière les potentialités offertes par l'environnement de formation, selon le sens qu'elles prennent dans son parcours, dans l'ordre de ses priorités et dans ses préoccupations du moment.

Ce cadre conceptuel permet de montrer que la place accordée aux objets techniques dans les dispositifs est plus sûrement révélatrice des modèles de l'apprentissage, sous-jacents aux conceptions pédagogiques que d'une fonction strictement utilitaire des objets. Il met également en valeur la relation de dépendance réciproque qui s'établit entre objet et activité, l'un pré-scrivant l'autre (selon le terme de M. Akhrich, 1987) et l'autre renforçant le caractère réificateur du premier.

\section{Types pédagogiques et modèles de l'apprentissage, structure des activités et fonctions des artefacts}

Une approche socio-historique du rapport aux objets techniques dans le domaine de la formation (Glikman, 2002; Moeglin, 2005) montre que chaque période a exploité les techniques du moment de manière essentiellement fonctionnelle, sans rompre pour autant avec la croyance implicite d'un lien causal d'efficacité directe entre la modernité des techniques et celle des pratiques. Aujourd'hui, la suprématie des perspectives économiques et l'abondance des discours ${ }^{8}$ de promotion contribuent à renforcer ces croyances. Pourtant, la complexification continue des environnements de travail associée à la croissance exponentielle des potentiels techniques, devrait au contraire conduire à une prise de distance critique et à une mutation profonde des modes d'action et de pensée.

En formation, il est particulièrement important d'identifier les modèles pédagogiques, explicites ou non, qui sous-tendent l'organisation des activités, ainsi que les relations entre conceptions de l'apprentissage et rapports aux artefacts. La démarche est nécessaire si l'on veut comprendre les raisons des nombreux décalages entre projets et résultats observés sur le terrain, et plus largement, les difficultés rencontrées par les pédagogies individualisantes de masse qui tentent de résoudre le paradoxe en hybridant, dans des proportions variées, des formes artisanales et industrielles ${ }^{9}$.

\section{Trois « types » de pédagogies}

Travaux théoriques ${ }^{10}$ dans le champ éducatif et enquêtes menées sur divers terrains de l'enseignement et de la formation, conduisent à distinguer trois grands « types » d'intervention pédagogique (au sens wébérien d'idéal-type): les pédagogies de la transmission centrées sur l'exposé magistral des contenus; les pédagogies de l'entraînement orientées vers le modelage comportemental; les pédagogies du développement qui privilégient l'accompagnement de l'activité des apprenants. Ces types sont définis par des indicateurs qui décrivent les finalités des projets, les modes d'organisation des environnements, ainsi que les rapports pratiques aux objets techniques qu'ils déterminent pour les étudiants (tableau 1, ci-dessous). Selon la modalité pédagogique privilégiée chaque type développe des objectifs, fonctions et méthodes qui relèvent plutôt de la transmission de contenus, de l'entraînement de compétences et de savoir-faire ou du développement personnel des processus d'apprentissage. Dans chaque cas, la structure et l'organisation des environnements imposent des formes reconnaissables d'articulation entre objets techniques et intervention humaine, que ce soit au plan de la mise en scène et de la représentation des contenus, des interactions formatives ou des relations entre utilisateurs et instruments. Ces structures qui proviennent de conceptions différentes du rapport à la connaissance, aux techniques et à la formation des personnes, induisent et renforcent des pratiques très différentes.

Dans les pédagogies de la transmission, l'organisation en présence ou à distance de l'environnement, est d'abord conçue en fonction de l'activité de l'instance de formation. Les artefacts sont utilisés en tant que supports et visent à concentrer l'attention des apprenants sur les contenus et les discours de référence. Les espaces de travail (salles de cours ou 
d'informatique, laboratoires multimédia, plateformes numériques) orientent leur action en référence à l'espace magistral (le « cours», première étape de la navigation, est différencié des " exercices d'application »). Le formateur détient l'essentiel des moyens du contrôle visuel (estrade, poste multimédia, écran de contrôle, traceur), auditif (code du silence, poste audio, bruitages) et symbolique (sanctions verbales et non-verbales, auditives ou iconographiques). C'est à lui que revient l'initiative des échanges, la planification des activités et en conséquence, le plus grand volume d'interventions.

Tout en poursuivant des objectifs d'individualisation, les pédagogies de l'entraînement ne modifient pas profondément ce schéma. Pour l'essentiel, les supports techniques offrent des activités très structurées, voire programmées, qui visent à faire acquérir des contenus, mais surtout à modifier des comportements ou des modes de raisonnement, à partir de progressions méthodiques. En fonction des parcours prédéfinis et des potentiels technologiques retenus (fonctions d'aide et d'autocorrection, analyses de réponses, feedbacks synchrones et asynchrones), l'entraînement des apprenants s'exerce sur une variété de savoir-faire dont certains s'enchaînent en montages complexes à visée d'expertise.

Les pédagogies du développement renversent les perspectives en focalisant la structuration des environnements sur les processus mêmes de l'apprentissage. La mise en scène et l'organisation des éléments (modes de présentation et d'exploitation des contenus, disponibilité des artefacts, interactions entre humains et machines, contrôle et évaluation) sont conçues pour favoriser le travail indépendant et inciter les sujets à prendre des initiatives. Les espaces ouverts (médiathèques, espaces de ressources matériels ou virtuels, salles de travail diversifiées) hébergent des objets, des actions et des modalités d'intervention empruntés à des contextes sociaux et culturels hors cadre institutionnel (littérature, conférences, presse et médias audiovisuels, web). Les unités de lieu (la classe), de temps (le cours) et d'action (l'enseignement) se trouvent disloquées par l'orchestration d'activités guidées ou autonomes, individuelles ou collectives.

Ces trois «types » de pédagogies se retrouvent sous des formes hybrides sur tous les terrains éduca- tifs. Ils n'en relèvent pas moins de modèles très différents et largement opposés de l'apprentissage, dont certains sont directement liés aux travaux de la psychologie.

\section{Les modèles sous-jacents de l'apprentissage}

Les pédagogies de la transmission dont le modèle magistral est le prototype s'appuient sur une conception traditionnelle, hiérarchique descendante. Elles supposent l'existence d'un savoir de référence consensuel, transmissible et mémorisable (Crahay, 1999). L'analyse des pratiques et la proposition de modèles ${ }^{11}$, notamment didactiques, lui apportent un caractère reproductible, généralisable et par conséquent économique, particulièrement attractif en situation de massification. Les limites du modèle magistral tiennent à l'affaiblissement de la stabilité culturelle et du consensus social qui le justifiaient jusque-là, mais aussi à la dissociation entre la transmission des connaissances et leur exploitation propre au schéma « leçon-application ». Si le modèle permet d'ordonner les contenus de manière générique selon une succession logique structurée et des exercices du simple au compliqué, il s'adapte mal en revanche aux différences individuelles et répond rarement à l'hétérogénéité croissante des profils et des besoins des publics contemporains.

En opposition explicite au primat cognitif et culturel du modèle magistral (Skinner, 1968), le modèle behavioriste s'appuie sur le postulat selon lequel il est possible d'étudier et de modeler directement les comportements sans rentrer dans la « boîte noire » des processus mentaux. Tout apprentissage peut s'obtenir par conditionnement simple en associant un stimulus d'entrée (input) à sa réponse comportementale (output), observable et contrôlable. La répétition et l'enchaînement des segments élémentaires de comportement en séquences assurent le renforcement et la progression ordonnée des acquisitions du simple au complexe. Le modèle behavioriste a permis le développement d'une science expérimentale de l'apprentissage en laboratoire, fondée sur la programmation rationnelle de comportements objectifs et mesurables. L' "enseignement programmé », versant pratique de ce modèle, a tenté de recréer artificiellement des conditions optimales d'apprentissage par la réduction et la progression rigoureuse des items, la neutralisation des perturbations cognitives 
et l'individualisation des rythmes. Le principe du retour immédiat d'information (feed-back) qui assure « renforcement positif » et motivation de l'apprenant, ainsi que la structuration de la progression ${ }^{12}$ à partir d'une analyse didactique ${ }^{13}$ méthodique des contenus, préparent la voie aux applications techniques.

Dans ses fondements, le modèle cognitiviste se différencie radicalement des travaux behavioristes en ce qu'il s'intéresse à la «boîte noire » mentale des individus (représentations, processus, raisonnements) qui traite la transformation interne des entrées perceptives en réponses rationnelles. À partir de l'analogie entre cerveau et ordinateur, le cognitivisme d'origine définit la connaissance comme un traitement logico-symbolique d'information. Il applique à l'apprentissage le schéma des trois stades de traitement associés à la physique de l'ordinateur classique: l'encodage (transcription en bits et symboles); le traitement symbolique (par calcul logique); le stockage (conservation en mémoire). Pendant trente ans, l'activité cognitive humaine a été ainsi assimilée à un système de traitement informationnel et l'apprentissage redéfini en conséquence. La psychologie cognitive s'est longtemps appuyée sur ce modèle pour formaliser et automatiser avec succès de nombreux processus d'action, de connaissance et d'apprentissage humains (Gardner, 1985), à condition de s'en tenir à des niveaux cognitifs restreints et abstraits (Linard, 1989). Elle s'est progressivement ouverte aux perspectives biologiques des neurosciences, mais elle continue à minorer l'importance des dimensions sociales et affectives, ainsi que les sources de différences interindividuelles de l'activité cognitive.

Le modèle behavioriste qui vise l'acquisition d'automatismes comportementaux et le cognitiviste qui assimile la connaissance au traitement symbolique d'informations prédéfinies, ont chacun leur secteur légitime d'application et d'optimisation. Leur limite en formation réside dans le fait qu'ils conduisent à privilégier les concepts, procédures et résultats déterminés par l'instance experte de formation, en ignorant les démarches et processus personnels des sujets, non experts, qui cherchent à les comprendre et à se les approprier.

Le modèle socioconstructiviste, héritier de la psychologie de l'intelligence (Vygotsky, Piaget, Wallon, Dewey, Bruner) renverse les perspectives et offre une alternative aux modèles précédents. Il ré-introduit la dimension biologique du corps, la sensorimotricité et le milieu, comme facteurs premiers de la genèse de l'intelligence. Les interactions avec le milieu, les dispositions individuelles et les interactions interpersonnelles deviennent autant de constituants déterminants de l'acquisition et de la production individuelle et collective de connaissance ${ }^{14}$. Celle-ci, définie à la fois comme processus et produit de l'activité intelligente, est le fruit de l'action délibérée d'un sujet qui s'auto-construit dans l'interaction avec son milieu - physique, social et symbolique - en réponse à un double besoin d'adaptation à l'environnement et d'intégration sociale. Le sujet est ainsi perçu dans une triple perspective biologique, psycho-affective et sociale. Contrairement aux modèles précédents, il n'y a pas de séparation entre acquisition de connaissances et mobilisation individuelle des démarches et des processus cognitifs qui les génèrent. Face à une situation nouvelle ou perçue comme "problème » (Piaget) à résoudre ou « enquête » (Dewey) à mener, le sujet agit toujours en fonction de son environnement naturel et social et en interaction avec lui (Vygotsky, Bruner). En formation, les limites de ce modèle résident dans la complexité de sa vision globale de l'apprentissage et dans les nombreuses conditions qu'exige sa mise en œuvre.

Lorsque l'on cherche à comprendre le rapport que les pratiques de formation entretiennent avec les artefacts et les modèles sous-jacents d'apprentissage, on observe diverses formes de combinaison, plus ou moins proches de l'une ou l'autre dominante (Albero, 1998). Contrairement aux modèles théoriques (magistral, béhavioriste, cognitiviste, socioconstructiviste) qui se définissent de façon tranchée, les pratiques de formation ont une approche plus opportuniste des différents modèles. Il reste à comprendre de quelle manière ceux-ci se juxtaposent ou s'articulent et quelle place y occupent les objets techniques.

\section{Fonction et rôles des artefacts}

Chaque type de pédagogie tend naturellement à privilégier les artefacts qui répondent le plus directement à ses objectifs mais, avec la généralisation du numérique, ce sont moins les supports que leurs usages qui différencient la mise en acte des modèles sous-jacents. Dans la perspective du couplage socio- 
technique, les modèles d'apprentissage sous-jacents aux types pédagogiques ne déterminent pas seulement la sélection des instruments, ils définissent également le choix de leurs fonctions et rôles, euxmêmes liés aux modes d'organisation de l'espacetemps des activités de formation.

Les pédagogies de la transmission qui dirigent l'activité des apprenants vers la mémorisation et l'application de textes et de savoirs de référence, privilégient les contenus informatifs et les cours médiatisés, les exercices écrits d'application ne comportant qu'une interactivité minimale. L'artefact assume ainsi une fonction d'auxiliaire dans une structure ordonnée de programme normatif dont les caractéristiques ne sont pas modifiées.

Les pédagogies de l'entraînement visent l'acquisition d'automatismes comportementaux (modèle behavioriste) ou de procédures cognitives d'analyse et de résolution de problème (modèle cognitiviste) privilégient les exerciseurs, tutoriels et simulateurs. Certains, à interactivité faible, présentent une variété de situations exemplaires en fonction de tâches prescrites. D'autres, au degré d'interactivité plus important, visent à susciter des processus cognitifs et métacognitifs plus complexes tels que l'analyse, la déduction et la réflexion sur les résultats. Dans ces modèles, les artefacts sont conçus comme des outils d'exercice et d'apprentissage d'unités prédéterminées. Ils sont intégrés dans un système qui conserve les caractères essentiels de la structure magistrale en y ajoutant une dimension dynamique par la prise en compte de certaines caractéristiques individuelles.

Les pédagogies du développement qui ont pour objectif l'autostructuration interne du sujet à partir de ses interactions avec l'environnement, recherchent la diversification des espaces et des méthodes de travail et d'exploration, d'aide et de communication. Elles offrent une palette de possibles qui vise à induire une interactivité importante. L'accompagnement incite chacun à choisir, sinon organiser, son parcours de formation selon un projet personnel, des objectifs cohérents et des critères individuels d'acceptabilité de sa performance. Les artefacts interactifs, proches des jeux électroniques, proposent des simulateurs sous la forme de micromondes qui permettent à l'apprenant d'expérimenter par lui-même une grande diversité de situa- tions ${ }^{15}$. L'artefact remplit donc des fonctions plus complexes, à la fois techniques et sémiotiques. Il est un instrument au service des dimensions symboliques (langage, codes, méthodes) et des processus individuels d'appropriation des apprenants. Dans ce cas, et à condition de réunir les moyens d'un fonctionnement adéquat, l'environnement prend la forme d'un dispositif ouvert, doté des propriétés spécifiques (cohérence, dynamique, adaptabilité, autorégulation) dues à l'intégration dans son fonctionnement des dimensions propres à l'activité des acteurs (finalités, projets, motifs, dispositions). Les priorités sont moins la conservation de la structure que l'adaptation stratégique à la diversité des publics et des milieux et la capacité d'évolution dans le temps.

Le choix et l'utilisation des artefacts dans chaque type pédagogique expriment et en même temps confortent le mode d'interaction qui correspond à son modèle sous-jacent d'apprentissage. On trouve toutefois davantage de combinaisons hybrides dans les dispositifs qui font appel à des formes indépendantes de travail, que ce soit en présentiel (médiathèques et centres de ressources) ou à distance (FOAD).

L'analyse proposée ci-dessus (synthétisée par l'annexe 1) se veut moins normative qu'épistémique et praxéologique. La question n'est pas de savoir quel est le meilleur modèle en soi, mais ce qu'il est préférable de développer en fonction des situations, des buts et des objectifs poursuivis. En ceci, l'intervention et la recherche en formation, comme toute action éducative, ne peuvent faire l'économie de l'attention aux finalités et aux valeurs (économiques, sociales, axiologiques) qui orientent de façon décisive l'ensemble des autres dimensions de l'activité.

\section{De l'usage des objets techniques aux questions du pour quoi}

Un défi majeur posé par les objets techniques contemporains est le rythme apparemment incontrôlable de leur développement et la puissance non mesurée de leur influence sur les activités humaines. Dans tous les domaines, le poids de l'innovation est tel que les exigences techniques prennent régulièrement le pas sur le souci des finalités, l'évaluation des résultats et la réflexion critique sur l'action. Les 
acteurs, soumis à une pression toujours plus forte, se trouvent sans autre choix que la consommation captive. Dans les institutions, les investissements considérables (financier, intellectuel, énergétique) consacrés au renouvellement et à la maîtrise des moyens techniques de réponse aux problèmes induisent inévitablement les recherches d'économies sur d'autres plans: réduction de l'intervention humaine, maintien de conceptions erronées et reproduction de procédures inefficaces qui ont l'avantage d'être connues ${ }^{16}$, plus économiques ou aisément applicables. Enfin, la domination des stratégies qui préfèrent l'urgent à l'essentiel, la tâche aux relations et l'hyperactivité au suivi de ses conséquences dans le temps, multiplie les effets pervers des réponses à court terme.

Ces tensions vont dans le même sens, souvent en contradiction avec les missions éducatives de la formation pour lesquelles le développement humain reste à la fois l'objectif, le moyen et l'indicateur de réussite. Les ressorts de l'enseignement, de l'apprentissage et de l'intégration sociale se trouvent donc doublement affectés par l'expansion des méthodes et des objets techniques: par la richesse de leurs potentiels et par les dérives de leur surconsommation. La compréhension large de l'ensemble des phénomènes et des possibles de l'action devient un enjeu plus important que jamais dans le domaine.

Au plan de la recherche, cette contribution tente de montrer que les perspectives élaborées en SHS permettent de renouveler profondément la compréhension des environnements de formation. Associée au couplage humains-artefacts, l'approche sociotechnique offre en particulier des bases théoriques et des outils d'analyse qui permettent une saisie plus compréhensive de la complexité des dispositifs actuels, à la fois produits et déterminants de l'action humaine ${ }^{17}$. Elle met en évidence l'interdépendance constitutive entre types de finalités pédagogiques et modèles d'apprentissage, conception des environnements, structuration de l'activité des acteurs et usage des objets techniques. Dans ce cadre, les discordances et les conflits observés sur le terrain ainsi que le caractère incertain des mesures et des résultats, même objectifs et quantifiés, n'apparaissent plus comme des accidents. Ce sont des dysfonctionnements, sinon normaux du moins inévitables, de l'action ordinaire ${ }^{18}$, liés aux tensions entre multiples dimensions, longtemps séparées ou ignorées par les découpages disciplinaires.

Au plan pratique, l'approche sociotechnique a également des incidences importantes. En élargissant l'analyse des environnements de formation aux logiques des acteurs et contextes ainsi qu'aux aléas de l'action, elle en rend la description plus fidèle mais aussi plus difficile. En revanche, le fait de reconnaître et d'intégrer dans l'analyse l'ensemble des phénomènes ${ }^{19}$ multiplie les moyens de les comprendre et de mieux remédier aux problèmes qui les accompagnent.

Une réflexion scientifiquement fondée sur les finalités et les conséquences de la technologisation généralisée des activités ${ }^{20}$ est d'autant plus pertinente que cette dernière fascine davantage. Comment ne pas s'interroger, par exemple, sur l'industrialisation massive de l'éducation et de la formation (Moeglin, 1998) et sa justification par les seuls critères technoéconomiques du marché et du management? Quelle place laisse-t-elle aux approches socio-relationnelles de type artisanal, nécessaires aux pédagogies du développement qui ne se soucient pas seulement de l'aspect rentable des connaissances mais de leur appropriation par les individus? Pourquoi, dans la conception des dispositifs, continuer à accorder autant d'attention aux ressources techniques et aux cahiers des charges des commanditaires et aussi peu aux capacités, conditions et besoins de ceux à qui ils sont destinés: ceci même quand expérience et études de terrain montrent qu'ils ne conviennent qu'à un faible pourcentage d'individus (moins de $20 \%$ selon Albero, Kaiser, 2009, 2010).

En tant que mode de relation au monde, la pensée et l'action technique ont depuis toujours suscité la réflexion des philosophes. Analysant l'excès du "pouvoir de faire » sur le "pouvoir de prévoir et de juger " propre aux technologies contemporaines, Jonas (1979) évoque la question de la responsabilité. Il prolonge celle posée en son temps par Heidegger (1954) à propos de la puissance illimitée d' "arraisonnement de la nature » et de la démesure engendrées par la technique moderne. En rapprochant les deux analyses, Linard $(2003 ; 2004)$ montre qu'elles sont plus actuelles que jamais pour la compréhension des enjeux de la modernité, dans les activités à visée sociale telle que de la forma- 
tion. La recherche dans ce domaine ne peut-elle (ne doit-elle?) pas contribuer à une forme particulière de veille et de régulation mettant en évidence les limites et les dégâts des réponses exclusivement techniques aux problèmes humains? Le chercheur précise son propos en empruntant à Searle (2004) l'opposition entre les deux principaux types de motivation qui animent l'action humaine: la motivation économique qui vise à créer et à répartir les biens à des fins d'intérêts particuliers et lamotivation déontique dont l'objectif est de définir les droits et devoirs en vue d'une régulation collective de ces fins au nom de l'intérêt général. Sauf à s'en remettre à la seule mécanique des rapports de force, la question du comment entraîne donc inévitablement celle du pour quoi (Linard, 2003, 2004.).
En période de grandes mutations, l'intérêt pour les questions de survie, d'efficacité ou de rentabilité l'emporte sur celui porté aux questions des valeurs et des finalités. Les secteurs sociaux à forte dimension déontique, tels que l'éducation et la formation, s'en trouvent marginalisés d'autant et peinent à faire entendre un discours humaniste. L'approche sociotechnique montre que c'est dans la prise en considération de toutes les dimensions interdépendantes de l'action des acteurs que réside finalement l'efficacité de l'activité humaine. Dans un tel contexte, le rôle des SHS n'est-il pas précisément de maintenir avec obstination les questions déontiques du «pour qui » et du «pour quoi» aux côtés des questions seulement fonctionnelles $\mathrm{du}$ « comment faire $» ?$ 


\section{NOTES}

1. Nous entendrons le terme «formation » dans son sens le plus générique: en tant qu'action externe finalisée de " mise en forme » d'un individu ou d'un collectif par une instance sociale et de processus interne d'apprentissage et d'actualisation de soi. Ainsi comprise, la formation peut se définir comme la systématisation d'une forme particulière de médiation sociocognitive à visée éducative, sous-tendue par des valeurs et des projets partagés.

2. Dites: nouvelles, éducatives, de l'éducation, de la formation, de l'information et de la communication, etc.

3. Entre centration sur l'instance de formation (enseignement programmé, EAO, EIAO, enseignement par correspondance, EAD) et centration sur l'action de l'apprenant (e-learning par exemple), les termes intermédiaires de FAD, FOAD et e-formation renvoient indifféremment à l'instance de formation et à l'apprenant. Selon la définition qui est accordée au terme formation, la centration sous-jacente se fait plutôt sur l'une (donner une forme à autrui) ou l'autre (se donner une forme) de ces figures.

4. « De artis factum, fait de l'art. Phénomène d'origine humaine, artificielle (extrait du Petit Robert). Par sa définition large venant de l'anthropologie, la notion d'artefact présente l'avantage de ne pas focaliser l'analyse de la situation pédagogique sur les seuls objets matériels.

5. Cela permet ainsi de comprendre qu'un ordinateur utilisé comme une machine a écrire est, de fait pour le sujet qui l'utilise de la sorte, une machine à écrire, quelles que soient les potentialités techniques de la machine. De même, les potentialités explicatives ou opératoires d'un concept ne sont actualisées qu'en fonction de l'univers épistémique de chaque sujet. L'aide méthodologique (auto-évaluation, conseils, références documentaires, etc.) ne sera exploitée par un apprenant qu'en fonction des représentations qu'il se fait de l'intérêt et du potentiel de cette aide.

6. Cet accompagnement peut être pris en charge par des artefacts divers: questionnaires sur les stratégies d'apprentissage, grilles d'auto-évaluation commentées, fiches de conseils, sketch télévisé, modèles à imiter, etc.

7. C'est ce qui peut être observé dans certains dispositifs de e-learning, très sophistiqués au plan technique mais conçus d'après une analyse essentiellement rationnelle des objectifs et des tâches largement indépendante des situations, des besoins et des demandes des usagers.

8. Discours issus des recherches à orientation scientifique, mais aussi discours d'experts (politiques, ingénieurs, chefs de projets et autres professionnels), témoignages de praticiens et analyses réflexives.

9. Les catégories « artisanal » et « industriel » sont empruntées à A. Leroi-Gourhan (1943, 1971). L'artisan, isolé ou groupé, est spécialiste à temps complet et maîtrise toute la chaîne de production. Sa technique est aussi un « art » dans la mesure où il développe un style et un savoir-faire particulier issu de son histoire, de sa formation, de ses dispositions, de son expérience. L'auteur montre comment l'organisation industrielle, en regroupant les individus de manière hiérarchique, rend les moyens d'action indépendants des exécutants. On peut voir cette logique à l'œuvre dans la séparation aujourd'hui de règle dans les dispositifs de formation entre les tâches de programmation, développement, diffusion des divers supports, celles d'administration et de gestion, et celles d'enseignements des disciplines, contrôle et validation. La séparation des tâches s'accentue avec la numérisation et la mise à distance (ingénierie de formation, ingénierie pédagogique design, scénarisation, développement des environnements numériques, maintenance technique, tutorat en ligne, etc.).

10. Pour une analyse des courants pédagogiques: Palmade (1953), Mialaret (1977; 1992), Resweber (1986), Houssaye (1993), Legros \& Crinon (2002).

11. Par exemple: Gagné, 1977; Malglaive, 1990; Mialaret, 1977; 1992.

12. Etapes de la programmation: définition des objectifs; analyse didactique; construction d'épreuves d'encadrement des performances par des pré- et post-tests; choix technique du type optimal de programme; rédaction des segments de cours, puis de chaque item; expérimentation (Leclerc et al., 1977).

13. L'analyse didactique comprend la définition des contenus et l'organisation des chapitres, la délimitation des notions à acquérir, leur progression et leur ordre d'apparition dans les chapitres (Leclerc et al., 1977).

14. Pour J. Piaget (1972) par exemple, si le savoir trouve son origine dans l'action pratique et si l'activité cognitive es analysée comme une action qui porte sur des objets symboliques (ibid., 1974), ce sont bien les 
processus de décentration et d'abstraction qui conditionnent le passage de l'acte pratique à l'acte sémiotique et au concept opératoire. Dans ce passage, l'interrogation réflexive du sujet est primordiale (ibid.).

15. Par exemple, la série des jeux Sims (SimCity qui permet de comprendre dans l'action virtuelle ce qu'est la gestion d'une ville et SimAnt qui met en scène des fourmis et leur milieu de vie) ou encore les séries plus contemporaines des Serious Games.

16. Par exemple: la croyance obstinée dans la corrélation entre production de « cours » et acquisitions des étudiants, entre " mise à disposition » et " utilisation », entre " utilisation » et " apprentissages effectifs », entre « niveau de diplômation » et « compétences effectives ».

17. Leroi-Gourhan, 1943, 1945, 1971; Castoriadis, 1975, 1978; Akrich, 1987, 1989; Scardigli, 1992 ; Latour, 1992, 1999.

18. Albero, Linard, Robin, 2009.

19. Pour mémoire: conséquences du principe de couplage entre techniques et activité humaine; dépendances réciproques entre conceptions et types de pédagogie, objets techniques et modèles sous-jacents de l'apprentissage; formes diverses des hybridations comportant toujours une dominante; influence réciproque de l'idéel, du vécu et du fonctionnel de référence sur la conception, le fonctionnement et l'évolution des dispositifs; articulation conceptuelle entre dispositif de formation, instrumentation des apprentissages et configurations d'activité individuelles et collectives des acteurs; différenciation radicale entre artefact et instrument; intégration des dimensions situationnelles, temporelles et aléatoires de l'activité.

20. Mumford, 1934, 1967; Jonas, 1979; Roqueplo, 1983; Ellul, 1954, 1988; Sclove, 1995; Sfez, 2002; Feenberg, 2004. 
ANNEXE 1

\begin{tabular}{|c|c|c|c|}
\hline $\begin{array}{c}\text { Types pédagogiques } \\
\text { d'après leurs finalités } \\
\text { et modèles sous-jacents } \\
\text { d'apprentissage }\end{array}$ & Artefacts privilégiés & $\begin{array}{l}\text { Fonctions et rôles } \\
\text { des artefacts }\end{array}$ & $\begin{array}{l}\text { Structuration de } \\
\text { l'espace-temps } \\
\text { des activités }\end{array}$ \\
\hline $\begin{array}{l}\text { Pédagogies } \\
\text { de la transmission } \\
\rightarrow \text { Centrées sur des contenus } \\
\text { et progressions prédéfinis } \\
\text { (programmes) }\end{array}$ & $\begin{array}{l}\text { Manuels, supports papier, } \\
\text { multimédia de textes et icono- } \\
\text { graphies de référence } \\
\text { Cours en ligne }\end{array}$ & $\begin{array}{l}\rightarrow \text { Présentation } \\
\rightarrow \text { Illustration }\end{array}$ & $\begin{array}{l}\text { Principe des trois unités de lieu } \\
\text { (la classe), de temps (le cours) } \\
\text { et d'action (l'enseignement) }\end{array}$ \\
\hline Modèle magistral & Degré d'interactivité: 0 & Auxiliaire & Structure \\
\hline $\begin{array}{l}\text { Pédagogies } \\
\text { de l'entraînement } \\
\rightarrow \text { Centrées sur des compé- } \\
\text { tences et savoir-faire } \\
\text { (référentiels) }\end{array}$ & $\begin{array}{l}\text { Manuels, supports papier, } \\
\text { multimédia } \\
\text { Exerciseurs, tutoriels simples } \\
\text { (modèle béhavioriste). } \\
\text { Degré d'interactivité: } 1 \\
\text { Tutoriels évolués, modélisa- } \\
\text { tions (modèle cognitiviste) }\end{array}$ & $\begin{array}{l}\rightarrow \text { Répétition plus ou moins } \\
\text { variée d'exercices fixés } \\
\rightarrow \text { Progression plus ou } \\
\text { moins prédéfinie d'activités } \\
\text { modélisées }\end{array}$ & $\begin{array}{l}\text { Principe des trois unités } \\
\text { appliqué en salle d'informati- } \\
\text { que comme en salle de cours } \\
\text { classique }\end{array}$ \\
\hline $\begin{array}{c}\text { Modèles behavioriste } \\
\text { et Cognitiviste }\end{array}$ & Degré d'interactivité: 2 & Outil & Système \\
\hline $\begin{array}{l}\text { Pédagogies } \\
\text { du développement } \\
\rightarrow \text { Centrées sur la construc- } \\
\text { tion par le sujet de connais- } \\
\text { sances, stratégies d'appren- } \\
\text { tissage et méta-compétences } \\
\text { situés (projet individuel) }\end{array}$ & $\begin{array}{l}\text { Ouvrages autres que manuels, } \\
\text { documentations authentiques, } \\
\text { tous artefacts autorisant la } \\
\text { manipulation, fabrication ou } \\
\text { création de connaissances par } \\
\text { l'étudiant } \\
\text { Outils d'exploration et de simu- } \\
\text { lation, d'aide et de communi- } \\
\text { cation, micromondes. }\end{array}$ & $\begin{array}{l}\rightarrow \text { Accompagnement par } \\
\text { aménagement de situations } \\
\text { et d'actions incitatives visant } \\
\text { l'apprentissage des moyens } \\
\text { d'apprendre }\end{array}$ & $\begin{array}{l}\text { Diversification des lieux, } \\
\text { temps, objets et modalités } \\
\text { d'apprentissage, incluant des } \\
\text { formes non conventionnelles }\end{array}$ \\
\hline Modèle socio-constructiviste & Degré d'interactivité: 3 & Instrument & Dispositif \\
\hline
\end{tabular}

Annexe 1: Les pédagogies et leurs artefacts 


\section{RÉFÉRENCES}

Akrich, M. (1989). La construction d'un système socio-technique. Esquisse pour une anthropologie des techniques. Anthropologie et Sociétés, 13 / 2, 31-54.

Akrich, M. (1987). Comment décrire les objets techniques? Techniques et cultures, 9, janvier-juin, 49-64.

Albero, B. (2010a, à paraître). Penser le rapport entre formation et objets techniques. Repères conceptuels et épistémologiques, dans G. Leclercq et R. Varga, Dispositifs de formation, quand le numérique s'en mêle. Paris : Hermès / Lavoisier.

Albero, B. (2010b, à paraître). De l'idéel au vécu : le dispositif confronté à ses pratiques, dans B. Albero, N. Poteaux, Enjeux et dilemmes de l'autonomie. Une expérience d'autoformation à l'université. Etude de cas. Paris : Les éditions de la Maison des Sciences de l'Homme, coll. praTICs.

Albero, B. (2010c, à paraître). La formation en tant que dispositif : du terme au concept, dans B. Charlier et F. Henri (dir. par), La technologie de l'éducation : recherches, pratiques et perspectives. Paris: PUF, coll. Apprendre.

Albero, B. (2004). Travaux, interrogations et pistes de réflexion dans un champ de recherche éclaté. Note de synthèse. Savoirs, Technologies et formation, 5, novembre, 11-69.

Albero, B. (2003a). L'autoformation dans les dispositifs de formation ouverte et à distance : instrumenter le développement de l'autonomie dans les apprentissages. Dans I. Saleh, D. Lepage, S. Bouyahi (coord. par), Les tic au cour de l'enseignement à distance (pp. 139-159), Actes de la journée d'étude du 12 novembre 2002, organisée par le Laboratoire Paragraphe, Université Paris VIII, coll. Actes Huit.

Albero, B. (2003b). L'instrumentation des apprentissages dans les dispositifs à finalité de formation, note de synthèse pour l'obtention de l'Habilitation à diriger des recherches en Lettres et Sciences humaines, présentée à l'Université Paris 7-Denis Diderot par le Pr. Baudouin Jurdant, le 18 décembre.

Albero, B. (1998, 2000). L'autoformation en contexte institutionnel : du paradigme de l'instruction au paradigme de l'autonomie. Paris : L'Harmattan, coll. Éducation et formation, série Références.

Albero, B., Kaiser, A. (2010, à paraître). La Formation à Distance sélectionne un public d'autodidactes. De la fatalité à la prise en compte des pratiques effectives, Savoirs. Publié en allemand : Blended Learning : Didaktische Grundlagen, empirische Befunden, praktische Konsequenzen, Grundlagen der Weiterbildung - Praxishilfen (GdW-Ph). 6.90.230, S. 1 - 23. Köln (Luchterhand).

Albero, B., Kaiser, A. (2009). Attitudes et préférences des usagers face à la FOAD : les leçons d'une enquête, Distance et Savoirs, 7/1, 31-37. Publié en allemand sous le titre : Kein Angebot für jedermann. BlendedLearning: Einstellungen und Präferenzen der Nutzer, Weiterbildung. Zeitschrift für Grundlagen, Praxis und Trends, 2, avril-mai 2008, 30-33.

Albero, B., Thibault, F. (2006). E-learning et enseignement universitaire en France, dans CRUI, CPU, FVU, Les universités européennes à l'heure du E-learning. Regards sur la Finlande, l'Italie et la France. Commission européenne, Direction générale pour l'enseignement et la culture - Initiative e-learning (cet ouvrage a également été publié en anglais sous le titre : University towards e-learning: a focus on Finland, France and Italy).

Albero, B., Thibault, F. (2004). Enseignement à distance et autoformation à l'université : au-delà des clivages institutionnels et pédagogiques? Dans I. Saleh et S. Bouyahi, Enseignement à distance : épistémologie et usages (pp. 35-52), Chapitre 2, Paris, Hermès Science / Lavoisier.

Albero, B., Linard, M., Robin, J-Y. (2009). Petite fabrique de l'innovation ordinaire à l'université. Quatre Parcours de pionniers. Paris : L'Harmattan, coll. Logiques sociales.

Barbier, J-M. (dir. par) (2000). L'analyse de la singularité de l'action. Paris : PUF, coll. Education et formation.

Barbier, J-M. (sous la dir. de) (1996). Savoirs théoriques et savoirs d'action. Paris: PUF, coll. Pédagogies d'aujourd'hui. 
Barbier, J-M., Durand M. (sous la dir. de) (2006). Sujets, activités, environnements. Approches transverses. Paris : PUF, coll. Education et formation.

Bateson, G. (1977). Vers une écologie de l'esprit, 2 tomes, (Steps to an Ecology of Mind). Paris : Seuil, coll. Recherches anthropologiques.

Castoriadis, C. (1978). Les carrefours du labyrinthe 1. Paris : Seuil.

Castoriadis, C. (1975). Technique, Encyclopadia Universalis, vol. 15, 803-809.

Compte, C. (1998). La rhétorique télévisuelle : une médiation pour l'apprentissage des langues, Les Cahiers du CIRCAV (Centre Interdisciplinaire de Recherche sur les Communications Audiovisuelles), 10. Université de Lille III : Gerico, 227-249.

Compte, C. (1985a). Using Soap Opera Structure for Aural French Comprehension, PhD. New York University.

Compte, C. (1985b). Professeur cherche document authentique en vidéo. Études de Linguistique Appliquée, 58, 43-53.

Compte, C., Minacori, P. (1998). Des stratégies pour traiter l'information télévisée. État d'une recherche. Revue Internationale d'Éducation, 18, Paris, CNDP.

Compte, C., Mouchon, J. (1984). Décoder le journal télévisé. Paris, BELC / CIEP.

Crahay, M. (1999). Psychologie de l'éducation. Paris : PUF, coll. Premier Cycle.

Ellul, J. (1988). Le bluff technologique. Paris : Hachette, coll. Sociologie Pluriel.

Ellul, J. (1954). La Technique ou l'Enjeu du siècle. Paris : Armand Colin.

Feenberg, A. (2004). (Re)penser la technique. Vers une technologie démocratique. Paris : La Découverte / MAuss, coll. Recherches.

Gagné, R.M. (1977). The conditions of learning. New York : Rinehart \& Winston.

Gardner, H. (1985, 1993). Histoire de la révolution cognitive. La nouvelle science de l'esprit, trad. par J-L. Peytavin (The mind's new science. A history of the cognitive revolution). Paris : Payot, coll. Bibliothèque scientifique.

Glikman, V. (2002). Des cours par correspondance au 'e-learning'. Paris : PUF, coll. Éducation et formation.

Houssaye, J. (sous la dir. de), 1993. La pédagogie : une encyclopédie pour aujourd'hui. Paris : ESF.

Jacquinot-Delaunay, G., Monnoyer, L. (coord. par) (1999). Le dispositif. Entre usage et concept. Hermès, 25, Paris, CNRS Éd.

Jonas, H. (1979, 1990). Le principe de responsabilité : une éthique pour la civilisation technologique, trad. par J. Greisch (Das Prinzip Verantwortung, Frankfurt, Verlag). Paris : Éditions du Cerf/ Flammarion.

Kuhn T.S. (1962, 1983). La structure des révolutions scientifiques, trad. L. Meyer (The Structure of Scientific Revolutions). Paris : Flammarion, coll. Champs.

Latour, B. (1999, 2001). L'espoir de Pandore. Pour une version réaliste de l'activité scientifique, trad. par D. Gille (Pandora's Hope. Essays on the Reality of Sciences Studies). Paris : La Découverte.

Latour, B. (1992). Aramis ou l'amour des techniques. Paris : La Découverte, coll. Textes à l'appui, Anthropologie des sciences et des techniques.

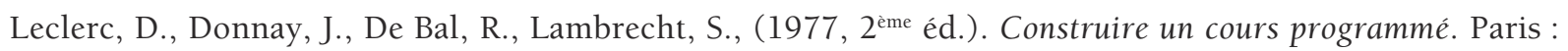
Nathan.

Legros, D., Crinon, J. (2002). Psychologie des apprentissages et multimédia. Paris : Armand Colin, coll. U Psychologie.

Leroi-Gourhan, A. (1943, 1971). L'Homme et la matière (Vol. 1 de Évolution et techniques). Paris : Albin Michel.

Leroi-Gourhan, A. (1943, 1945). Évolution et techniques, 2 volumes. Paris : Albin Michel.

Linard, M. (2004). Une technologie démocratique est-elle possible ? Savoirs, 5, 73-78. 
Linard, M. (2003). Autoformation, éthique et technologies : enjeux et paradoxes de l'autonomie. Dans B. Albero (sous la dir. de), Autoformation et enseignement supérieur (pp. 241-263). Paris : Hermès / Lavoisier.

Linard, M. (1989, 1996, 2 $2^{\text {ème }}$ éd. réactualisée). Des machines et des hommes. Apprendre avec les nouvelles technologies. Paris : L'Harmattan, coll. Savoir et formation.

Malglaive, G. (1990). Enseigner aux adultes. Paris : PUF, coll. Pédagogie aujourd'hui.

Marks Greenfield, P. M. (1984). Mind and Media. W. Collins Sons \& Co. Ltd.

Mialaret, G. (1992). Pédagogie générale. Paris : PUF.

Mialaret, G. (1977). Traité des sciences pédagogiques. Paris : PUF.

Moeglin, P. (2005). Outils et médias éducatifs. Une approche communicationnelle. Grenoble : PUG.

Moeglin, P. (sous la dir. de) (1998). L'industrialisation de la formation. État de la question. Paris : CNDP, coll. Documents, actes et rapports pour l'éducation.

Mumford, L. (1967, 1973). Le mythe de la machine, 2 vol., trad. par L. Dilé (The Myth of the Machine). Paris : Fayard, coll. Le phénomène scientifique.

Mumford, L. (1934, 1950). Technique et civilisation, trad. par D. Moutonnier (Tecnics and Civilization). Paris : Seuil.

Neisser, U. (1967). Cognitive psychologie. New York : Appleton Century Crofts.

Noble, D.F. (2001). Digital Diploma Mills. The Automation of Higher Education. New York : Monthly Review Press.

Palmade, G. (1953, 1991, 14 ème éd.). Les méthodes pédagogiques. Paris : PUF.

Perriault, J. (1996). La communication du savoir à distance. Autoroutes de l'information et télé-savoirs. Paris: L'Harmattan, coll. Éducation et Formation, série Références.

Perriault, J. (1989). La logique de l'usage. Essai sur les machines à communiquer. Paris: Flammarion.

Piaget, J. (1974). Comprendre et réussir. Paris : PUF.

Piaget, J. (1972). La prise de conscience. Paris : PUF.

Rabardel, P. (1995). Les hommes et les technologies. Approche cognitive des instruments contemporains. Paris: Armand Colin.

Resweber, J-P. (1986). Les pédagogies nouvelles. Paris : PUF.

Roqueplo, P. (1983). Penser la technique. Pour une démocratie concrète, Paris, Seuil, coll. Science ouverte.

Roublot, F. (2010, à paraître). Analyse des trajectoires d'activité des étudiants. Dans B. Albero, N. Poteaux, Enjeux et dilemmes de l'autonomie. Une expérience d'autoformation à l'université. Etude de cas. Paris: Les éditions de la Maison des Sciences de l'Homme, coll. PraTICs.

Salomon, G. (ed.) (1993). Distributed cognitions. Psychological and educational considerations. Cambridge: University Press.

Scardigli, V. (1992). Les sens de la technique. Paris : PUF, coll. Sociologie d'aujourd'hui.

Sclove, R. (1995, 2003). Choix technologiques, choix de société, trad. par I. Jami (Democracy and Technology). Paris : Ed. Charles Léopold Mayer, Descartes et Cie, coll. TechnoCité.

Searle, J.R. (2004). Liberté et neurobiologie. Réflexions sur le libre arbitre, le langage et le pouvoir politique. Paris : Grasset, coll. Nouveau Collège de Philosophie.

Sfez, L. (2002). Technique et idéologie. Un enjeu de pouvoir. Paris : Seuil, coll. La couleur des idées.

Simondon, G. (1958, 1969, 1989). Du mode d'existence des objets techniques. Paris : Aubier, coll. Philosophie. 